\title{
Promoção da saúde, qualidade de vida e iniquidade em saúde: reflexões para a saúde pública.
}

\section{Health promotion, quality of life and health inequity: reflections for public health.}

\section{Promoción de lasalud, calidad de vida y desigualdad en salud: reflexiones sobre lasalud pública.}

Aline Rodrigues de ALMEIDA ${ }^{1}$ Filipe Tadeu Santanna ATHAYDE ${ }^{2}$

\begin{abstract}
RESUMO: A ideia de promover saúde tem se tornado uma energia fundamental no movimento de saúde coletiva, no qual saúde é um fenômeno social e marcado por iniquidades. Este trabalho teve como objetivo compreender as relações entre promoção da saúde e iniquidades de acesso e assistência em saúde, identificandoos entraves para uma prática equânime da saúde pública com ênfase à qualidade de vida. Os diferentes perfis de doença e suas mediações sociais ultrapassam os estudos de variação biológica e incorpora o foco na dimensão social das vulnerabilidades. Para promover saúde no Brasil, é indissociável do enfrentamento de uma realidade de iniquidades históricas de grandes proporções que impõem desafios cotidianos não apenas ao setor de saúde, mas a todos aqueles que constroem políticas públicas. A qualidade de vida depende da satisfação das necessidades básicas de todos os cidadãos, propondo uma gestão baseada na solidariedade social, uma visão holística dos problemas e a redução das iniquidades. A promoção da saúde em um país tão desigual como o Brasil propõe um desafio constante aos atores envolvidos no sistema de saúde. A compreensão das iniquidades em saúde para estabelecer uma assistência holística é um processo difícil, mas extremamente relevante para a saúde pública.
\end{abstract}

Palavras-chave: promoção da saúde; qualidade de vida; equidade em saúde.

ABSTRACT: The idea of health promoting has become a major power in the movement of public health, on which health is a social phenomenon and marked for inequities. This work aimed to understand the relationship between health promotion and inequalities of access and health care, by identifyingthe barriers to an equal public health practice with emphasis on quality of life.The different profiles of disease and its social mediation beyond the studies of biological variation and incorporates a focus on the social dimension of vulnerability. To promote health in Brazil, is

1 Enfermeira. Especialista em Saúde Coletiva pelo Instituto de Educação Continuada, da Pontifícia Universidade Católica de Minas Gerais.Instituto de Educação Continuada, Pontifícia Universidade Católica de Minas Gerais.ninarodral@gmail.com

2 Professor do Instituto de Educação Continuada da Pontifícia Universidade Católica de Minas Gerais. Fisioterapeuta. Mestre em Ciências da Reabilitação pela Universidade Federal de Minas Gerais.ftsathayde@yahoo.com.br 
inseparable from facing a reality of major historical inequities that impose daily challenges not only to the health sector, but to all those who build public policies. The quality of life depends on the satisfaction of basic needs of all citizens, proposing a management based on social solidarity, a holistic view of the problems and reducing inequities. The health promotion in a country as unequal as Brazil proposes a constant challenge to the actors involved in the health system. The understanding of health inequalities to establish a holistic care is a difficult process, but extremely relevant to public health.

Keywords:health promotion;quality of life; equity in health.

RESUMEN: La idea de promoción de lasalud se ha convertido en una potencia importante en elmovimiento de la salud pública, enel que la salud es un fenómeno social y marcado por las desigualdades. Este trabajotuvocomo objetivo comprenderlarelación entre lapromoción de lasalud y las desigualdades de acceso y asistencia de lasalud, la identificácion de barreras para una práctica de lasalud pública de igualdad conénfasis en lacalidad de vida. Los diferentes perfiles de la enfermedad y su media ción social másallá de losestudios de variación biológica e incorpora un enfoque enladimensión social de la vulnerabilidad. Para promover lasaluden Brasil, es inseparable de frente a una realidad de grandes desigualdades históricas que imponendesafíosdiarios no sólo al sector de lasalud, sino a todos los que construyenlas políticas públicas. La calidad de vida depende de la satisfacción de las necesidades básicas de todos losciudadanos, proponiendo una gestión basada en la solidaridad social, una visión integral de los problemas y lareducción de las desigualdades. La promoción de lasaludenun país tan desigual como Brasil proponeundesafíoconstante para losactores involucrados enel sistema de salud. La comprensión de las desigualdades ensalud para establecer una atención integral es un proceso difícil, sino extremadamente relevante para lasalud pública.

Palabrasclave:promoción de lasalud; calidad de vida; equidadensalud.

\section{INTRODUÇÃO}

A ampliação da compreensão de saúde, como completo bem estar físico, mental e social e não somente ausência de doença tem sidolargamente discutida. Por mais adequada que a definição esteja o seu sentido prático, teórico e epistemológico não são bem compreendidos pelos profissionais de saúde, que ainda são guiados pelo modelo reducionista da assistência médica, abordando a causa, diagnóstico e tratamento da doença e não considerando o ambiente físico e o social onde osproblemas ocorrem ${ }^{1}$.

Saúde condiz com qualidade de vida e essa ideia precisa ser elucidada ${ }^{2}$. Aqualidade de vida somente é alcançada quando se oferece as condições mínimas para o desenvolvimento completo das possibilidades humanas como viver, sentir, amar, trabalhar, produzindo bens e serviços, fazendo ciências ou artes. Falta o esforço de fazer dessa temática algo mais concreta e operacionalizável ${ }^{3}$. 
A saúde como meta social e dos cuidados primários com a saúde foram tratados pela Conferência Internacional de ALMA ATA ${ }^{4}$. Com o objetivo de propor metas mundiais a conferência abordou assuntos sobre a atenção básica e as influências socioeconômicas, culturais, políticas na saúde e a importante estratégia de participação da sociedade para construção da promoção da saúde e prioridade de tratamento aos mais necessitados. Todos os elementos mais tarde foram incorporados aos programas de promoção da saúde, preconizados pela Política Nacional de Promoção da Saúde 5,6 $^{5}$

Promoção da saúde foi o nome dado a um processo de capacitação da população para atingir bem estar individual e coletivo com propósito de melhorar a qualidade de vida e saúde. Ainda existem dificuldades de compreensão para distinção dos conceitos de prevenção, educação em saúde e promoção da saúde. Compreender os conceitos facilita a elaboração de estratégias para a efetivação da prática de promoção da saúde. A prevenção, por sua vez, fundamenta-se e restringeseaorisco ou probabilidade de adoecer; enquanto a educação para a saúde é a transmissão e troca de conhecimentos com objetivo de estimular a prática de hábitos saudáveis, mudança de comportamento em que o indivíduo possa a ser responsável pelo seu estado de saúde $e^{5,6,7}$.

Nos últimos anos a promoção da saúde passou a ser apreendida como forma promissora de enfrentamento dos vários problemas que comprometem as populações. A partir de uma concepção ampla do processo saúde-doença e de seus determinantes, propõe a articulação de saberes científicos e populares, mobilização de recursos, programas estratégicos para seu enfrentamento e resolução ${ }^{8}$.

A equidade é um dos princípios fundamentais norteadores do Sistema Único de Saúde (SUS) no Brasil. Na legislação (Lei Orgânica da Saúde 8080) fala-se de "igualdade de assistência" como sinônimo de equidade ${ }^{9}$. No entanto, as práticas de saúde para a coletividade podem se modificar frente àdiversidade das necessidades da população. $\mathrm{O}$ esclarecimento sobre a importância de tratar os indivíduos diferentesde forma desigual para se alcançar a saúde é um desafio para gestores e profissionais da saúde, visto que o Brasil é uma dasnaçõesmais desiguaisdo planeta ${ }^{10}$.

As estratégias de promoção da saúde condizem intimamente com o constructo da qualidade de vida,além de requerer um olhar consciente do profissional da saúde frente ao contexto de iniquidades. A consciência das limitações do enfoque individual e a análise ampliada dos problemas sociais que interferem na saúde estimulamações efetivas em nível coletivo ${ }^{11}$.

A saúde pública se realiza por meio de políticas efetivas que atendam às necessidades da população nos campos da prevenção de agravos, promoção da saúde e medicina clínica. A promoção da saúde é a estratégia central para medicina social e objetiva oferecer qualidade de vida para a comunidade. A equidade é um pilar que deve ser considerado para efetivar as práticas de saúde assistindo os indivíduos de forma desigual para igualar a assistência ${ }^{12}$. Diante do exposto, o presente trabalho teve como objetivo compreender as relações entre promoção da saúdee iniquidades de acesso e assistência em saúde,identificando os entravespara uma prática equânime da saúde públicacom ênfase à qualidade de vida. 


\section{MÉTODO}

Trata-se de um estudo de revisão de literatura, em que realizou-se uma busca nas seguintes basesde dados eletrônicas: Literatura Latino Americana e do Caribe em Ciências da Saúde (LILACS), Literatura internacional em Ciências da Saúde (MEDLINE) e Base de Dados de Enfermagem (BDENF), além da Biblioteca Virtual da Universidade de São Paulo.

Foram utilizados, para busca dos artigos, os descritores: promoção da saúde, equidade, políticas de saúde, saúde pública, desigualdades em saúde, justiça social, qualidade de vida e iniquidade em saúde. Foram incluídos estudos publicados no período de 1994 a 2014, nos idiomas português e inglês, oriundos de pesquisa em bases de dados.No entanto, documentos clássicos, a maior parte de publicação mais antiga, foram adicionados de modo isolado por se tratarem de material de suma relevância para a construção da saúde coletiva e sustentação das discussões. Estes documentos consagrados foram: a Carta de Ottawa (1986), a Declaração da ConferênciaInternacional sobre Cuidados Primários de Saúde(ALMA-ATA, 1978), a Lei Orgânica da Saúde no Brasil,a Política Nacional de Promoção da Saúde e a Constituição da República Federativa do Brasil (1988).

Ao final da revisão, foram encontrados um total de 38 artigos, além dos documentos tradicionais. Após a leitura e análise detalhada foram excluídos 26 artigos por não condizerem com o objetivo proposto.Posteriormente, os 12 artigos restantes foram lidos na íntegra e compuseram a análise reflexiva.

\section{RESULTADO E DISCUSSÃO}

No Brasil, o Sistema Único de Saúde, resultado de embates políticos e ideológicos, refere-se à saúde como direito universal de cidadania, resultado das condições de saúde, educação, ambiente e trabalho ${ }^{13}$. Na Constituição Federal, artigo 196, refere que o acesso da população às ações e serviços para promoção, proteção e recuperação da saúde deve ser universal e igualitário ${ }^{14}$.

A carta da Primeira Conferência Internacional sobre Promoção da Saúde (Ottawa) foi um documento que fundamentou a promoção da saúde e a definiu como um trabalho de capacitação da comunidade para atuar na melhoria da qualidade de vida e da saúde ${ }^{7}$. A promoção da saúde, conforme preconizado pelo poder público brasileiro, implica em um processo abrangente e ininterrupto que envolve prevenção e educação e a participação de diferentes setores da sociedade na elaboração de estratégias que permitam a efetividade da educação para a saúde, transcendendo às decisões individuais. O desafio do SUS consiste em propor uma política transversal, integrada e intersetorial, que faça dialogar as diversas áreas do setor sanitário, os outros setores do Governo, os setores privados e não-governamental e a sociedade, compondo redes de compromisso e coresponsabilidade quanto à qualidade de vida da população ${ }^{5,6}$. 
As estratégias de promoção da saúde influenciamelementos comportamentais e culturais que são sabidamente nocivos à saúde e também estimula aqueles que conhecidamente contribuem para a permanência do ser saudável. O padrão comportamental e os hábitos de vida não seriam, portanto produtos isolados do meio em que se encontram, tampouco seriauma escolha descontextualizada ${ }^{2}$.

Entender os diferentes perfis de doença e suas mediações sociais ultrapassam os estudos de variação biológica e incorpora o foco na dimensão social das vulnerabilidades. $\mathrm{O}$ aumento do rendimento financeiro, por exemplo, possivelmente terá efeito na melhoria da saúde dos mais pobres ou que pessoas com baixa escolaridade teriam mais resistênciaàs ideias de prevenção de doenças. Estas condições sociais parecem ter profundo impacto sobre a saúde, evidenciando a importância de sua compreensão para direcionar as práticas assistenciais ${ }^{15}$.

A promoção da saúde no Brasil compartilha dos mesmos desafios de uma realidade de problemas sociais de grandes proporções que impõem desafios cotidianos não apenas ao setor de saúde, mas a todos aqueles que constroem políticas públicas ${ }^{16}$. O desenvolvimento de políticas econômicas eficientes é muito importante, uma vez que desemprego e baixos salários podem gerar incapacidade da família em adquirir os bens básicos para a sobrevivência, desencadeando fatores poderosos de adoecimento e morte, especialmente para crianças e idosos ${ }^{2,15}$.

Em países desenvolvidos e em desenvolvimento, os ricos vivem mais e ficam menos doentes que os pobres. A baixa escolaridade e o desemprego constituem grupos mais vulneráveis para problemas de saúde ${ }^{17}$. No Brasil os indivíduos mais pobres apresentam, por exemplo, uma pior condição de saúde bucal, o que tem sido associado a fatores como educação, renda e grupo racial ${ }^{18}$. As desigualdades entre as pessoas podem ocorrer por diversosmotivos, desde o padrão genético até os hábitos adquiridos no cotidiano ${ }^{19}$, no entanto, as iniquidades dizem respeito às disparidades que são criadas por um processo histórico e pelos modos de organização e de produção da sociedade ${ }^{20}$. Parece, então, que o estado de saúde e uma promoção de saúde efetiva estão intimamente ligadas aos aspectos sociais de uma população, sendo o acesso e a qualidade de assistência em saúde determinantes.

Para se construir as políticas geradoras de equidade,através dadistribuição de recursos, se faz com aumento da renda per capta e distribuição igualitária de renda. Juntamente com políticas que estimulem o crescimento econômico e diminuam a desigualdade, são estratégias que concedem uma sociedade mais justa ${ }^{21}$.

As iniquidades em saúde, além de sistemáticas e impactantes, também são evitáveis, injustas e desnecessárias. Os importantes avanços do conhecimento técnico-científico podem auxiliar na compreensão das iniquidades em saúde e, assim, os atores envolvidos poderiam colaborar na execução de práticas voltadas à promoção e à proteção da saúde coletiva ${ }^{12}$. A qualidade de vida depende da satisfação das necessidades básicas de todos os cidadãos, propondo uma gestão baseada 
na solidariedade social, uma visão holística dos problemas e a redução das iniquidades ${ }^{22}$.

A qualidade de vida é uma percepção humana, que tem sido aproximada ao grau de satisfação encontrado nas relações familiares e amorosas, social e ambiental e aopróprio caráter existencial do indivíduo ${ }^{1}$.Parao mundo ocidental, qualidade de vida representa o modelo de sociedade urbanizada, rica, valorizandoo conforto, prazer, boa mesa, moda, viagem, carro, televisão, telefone, computador, consumo de arte e cultura, entre outras comodidades e riquezas. Sociedades fortemente desiguaistendem a compreender o bem estar como o alcance de um padrão social superior ${ }^{23}$.

\section{CONCLUSÃO}

A ideia de promover saúde em um país tão desigual como o Brasil propõe um desafio constante aos atores de saúde. Compreender as iniquidades em saúde para estabelecer o cuidado é um passo difícil, mas extremamente relevante para a saúde coletiva. As políticas de enfrentamento das iniquidades que visam à redução da pobreza e propostas de atendimento de saúde podem garantiruma maior efetividade de ações de promoção da saúde e, consequentemente,melhor qualidade de vida.

As desigualdades sociais e as iniquidades em saúde para além da preocupação teórica e prática,constituem um problema ético. Ao classificar como injustas as causas sociais que levam ao adoecimento e à morte, evidencia-se a necessidade urgente de transformação. Portanto, a tarefa de superação deste paradigma passa necessariamente pela atuação política e de toda sociedade, sobretudopor aqueles cujos direitos estão constantemente sendo negados.

Para que as políticas de saúde se consolidem atendendo o interesse público e a promoção da equidade é necessário o fortalecimento do processo democráticomultiplicando os atores envolvidos instrumentando sua participação com o acesso à informação e conhecimento pertinente que permitam a defesa de seus interesses.

\section{REFERÊNCIAS BIBLIOGRÁFICAS}

1.Minayo MCS, Hartz ZMA, Buss PM. Qualidade de vida e saúde: um debate necessário. Ciência e Saúde Coletiva. 2000:5(1):7-18.

2. Buss PM. Promoção da saúde e qualidade de vida. Ciência da Saúde Coletiva. 2000:5(1):163-177.

3. Ruffino NA. Qualidade de vida: compromisso histórico da epidemiologia. Saúde Debate. 1994:(35):63-7.

4. Organização mundial de saúde. Atenção primária de saúde. Conferência internacional sobre atenção primária de saúde. Alma-Ata, 1978, URSS, 6-12. 
5. Souza EM, Grundy E. Promoção da saúde, epidemiologia social e capital social: interrelações e perspectivas para a saúde pública. Cad. Saúde Pública. 2004:20(5):1354-1360.

6. BRASIL. Ministério da Saúde. Secretaria de Vigilância em Saúde. Secretaria de Atenção à Saúde. Política Nacional de Promoção da Saúde. 3. ed. Brasília: Ministério da Saúde, 2010.

7. Organização mundial de saúde. Primeira conferência internacional sobre promoção da saúde. Carta de Ottawa, 1986, Canadá.

8. Buss PM, Carvalho AI. Desenvolvimento da promoção da saúde no brasil nos últimos vinte anos (1988-2008). Ciência e Saúde Coletiva. 2009: 14(6): 2305-2316.

9. BRASIL. Lei 8.080 de 19 de setembro de 1990. Lei Orgânica da Saúde. Brasília, 19 set. 1990.

10. Buss PM, Pellegrini Filho A. A saúde e seus determinantes sociais. Rev Saúde Coletiva. 2007: 17(1): 77-93.

11. Barata RB. Iniquidade e saúde: a determinação social do processo saúde-doença. Revista USP. 2001: n.15, 138-145.

12. Pellegrini Filho A. Pesquisa em saúde, política de saúde e equidade na américa latina. Ciência e Saúde Coletiva. 2004:9(2):339-350.

13. Sperandio AMG, Correa CRS, Serrano MM, Rangel HA. Caminho para a construção coletiva de ambientes saudáveis - são Paulo, brasil. Ciência e Saúde Coletiva. 2004:9(3):643654.

14. BRASIL. Constituição (1988). Constituição da República Federativa do Brasil. Brasília, DF, Senado, 1998.

15. Magalhães R. Monitoramento das desigualdades sociais em saúde: significados e potencialidades das fontes de informação. Ciência e Saúde Coletiva. 2007:12(3):667-673.

16. Campos GW, Barros RB, Castro AM. Avaliação de política nacional de promoção da saúde. Ciência e SaúdeColetiva. 2004:9(3):745-749.

17. WHO Europe. Social Determinants of health: The Solid Facts. Copenhagen: WHO; 2003.

18. Guiotoku SK, Moysés ST, et al. Iniquidades raciais em saúde bucal no brasil. Ver Panam Salud Publica. 2012:31(2):135-41.

19. Coelho IB. Democracia sem equidade: um balanço da reforma sanitária e dos dezenove anos de implantação do sistema único de saúde no brasil. Ciência e Saúde Coletiva. 2010:15(1):171-183.

20. Thyry-Cherques HR. John rawls: economia moral da justiça. Revista Sociedade e Estado. 2011:26(3):551-563. 
21. Barros RP, Henriques R, Mendonça R. Desigualdade e pobreza no brasil: retrato de uma estabilidade inaceitável. Rev Brasileira de Ciências Sociais. 2000:15(42):123-142.

22. Mendes EV. Município saudável: pra quê? Como? Seminário Nacional sobre Municípios Saudáveis. Faculdade de saúde pública da USP. 1999:13pp

23. Rocha AD, Okabe I, Martins MEA, Machado PHB, Mello TC. Qualidade de vida, ponto de partida ou resultado final? Ciência e Saúde Coletiva. 2000: 5(1):63-81.

Artigo apresentado em 23-10-14

Artigo aprovado em 02-08-15

Artigo publicado no sistema em 26-09-15 